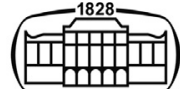

AKADÉMIAI KIADÓ

\section{Acta Veterinaria Hungarica}

$68(2020) 2,207-211$

DOI:

$10.1556 / 004.2020 .00020$

(c) 2020 Akadémiai Kiadó, Budapest

\section{ORIGINAL ARTICLE}

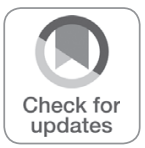

"Corresponding author.

Tel.: +4366460257 6455,

E-mail: peter.csebi@vetmeduni.ac.at

\title{
The slope of the pelvis is variable in dogs but does not show correlation with femoral subluxation
}

\author{
PÉTER CSÉBI $^{1 *}$, ALEXANDER TICHY $^{2}$, \\ MICHAELA GUMPENBERGER ${ }^{1}$ and EBERHARD LUDEWIG $^{1}$ \\ ${ }^{1}$ Department/Hospital for Companion Animals and Horses, Diagnostic Imaging, University of \\ Veterinary Medicine Vienna, Veterinärplatz 1, Vienna, 1210, Austria \\ ${ }^{2}$ Department of Biomedical Sciences, University of Veterinary Medicine Vienna, Vienna, Austria
}

Received: October 3, 2019 • Accepted: March 5, 2020

Published online: September 4, 2020

\section{KEYWORDS}

dog, canine hip dysplasia, Norberg angle, pelvic slope, femoral subluxation

\section{INTRODUCTION}

The pelvic limb has an important function in the propulsion of the body (Nickel et al., 1986). The differences in the slope of the pelvis and the other joint angulations influence the performance of the hind limbs. Steep pelvis conformation indicates a short back reach and it is beneficial for uphill running and quick-turning motion, while a flat pelvis indicates an extended back reach facilitating fast galloping (Gilbert and Brown, 2001).

Canine hip dysplasia (CHD) is defined as abnormal development of the hip joint, which usually results in coxarthrosis. The condition is due to several different factors including hereditary and environmental components (Kealy et al., 1992; Lust, 1997). Laxity of the hip joint is considered to be the main contributing factor to the development of CHD in an individual animal (Smith et al., 1995).

To define the cause of $\mathrm{CHD}$, several studies investigated the morphological parameters of the pelvis and the femur concerning hip dysplasia. The inclination angle of the femoral head and neck in different breeds showed only a minimal (nonsignificant) difference between dysplastic and nondysplastic dogs of seven breeds (Sarierler, 2004). A recent genetic and morphological study measured 13 pelvic dimensions, and it was concluded that the shape of the pelvis alone was not strongly associated with CHD (Fealey et al., 2017). This study did not investigate the sacroiliac joint or the lumbosacral junction. However, morphological changes in the sacroiliac joint may play a role in the development of $\mathrm{CHD}$, because the presence of an asymmetrical lumbosacral transitional vertebra favours pelvic rotation over its long axis, resulting in inadequate femoral head coverage by the acetabulum on one side. Inadequate 
coverage of the femoral head favours subluxation, malformation of the hip joint, and secondary osteoarthritis (Flückiger et al., 2017).

The aims of the current study were to compare the slope of the pelvis in different breeds of dogs and to test the hypothesis that the slope of the pelvis influences the degree of femoral subluxation, which is an important factor in CHD.

\section{MATERIALS AND METHODS}

\section{Study design and subject selection}

A retrospective study design was used. A second-year diagnostic imaging resident (P.C.) searched the archive of the authors' hospital for data produced between 2013 and 2018. Inclusion criteria were dogs over 12 months of age with symmetrically positioned digital ventrodorsal extended hip radiographs following the requirements for official hip dysplasia screening as well as symmetrically positioned lateral lumbosacral radiographs. These lumbosacral radiographs were available together with the hip radiographs because in Austria the hip screening protocol includes also lumbosacral transitional vertebra screening in several breeds. Our purpose was to investigate the pure effect of the slope of the pelvis on the hip joint; therefore, dogs with a transitional lumbosacral vertebra were not included in the study. For statistical reasons only breeds with at least 20 available dogs according to the inclusion criteria were included in the study. A total of 180 dogs from six different breeds were available for the investigation. The breeds of the dogs were Hungarian Vizsla $(n=24)$, Rottweiler $(n=30)$, Labrador Retriever $(n=40)$, Large Münsterländer $(n=30)$, Weimaraner $(n=34)$ and White Shepherd $(n=22)$. The number of female dogs $(n=107)$ was higher than that of male dogs $(n=73)$.

\section{Measurements}

The measurement of the Norberg angle (NA) was performed in standard extended ventrodorsal hip radiographs without rotation of the pelvis (Flückiger, 2007). A Dicom viewer software (dicomPACS ${ }^{\circledR}$ vet, version 8.9.5, 2018 Oehm und Rehbein $\mathrm{GmbH}$ ) was used for the calculations. The NA was calculated by measuring the angle between a line that connects the centre of the femoral head between the left and right hips and a line that connects the centre of the femoral head with the lateral tip of the cranial acetabular rim (Henricson et al., 1966). The NA was always measured in both hip joints, but only the smaller was recorded and used in the study.

The slope of the pelvis is defined as the angulation between the vertebral column and the axis of the body of the ilium. Since this angulation might be influenced by the positioning of the dogs for the radiological study, we created the more consistent and reproducible sacrum-pelvis angle (SPA), the angulation between the sacrum and the axis of the body of the ilium to represent the differences in the slope of the pelvis in our study. The SPA was measured on standard lateral lumbosacral radiographs with the same software application as the NA measurements. First, a circle was drawn so that the cranial margin of the circle superimposed on the contour of the cranial border of the more cranially located acetabulum. Then a second circle was drawn more cranially. The margin of this circle tangentially contacted the iliac crest dorsally and the ventral aspect of the ilial body. The centre point was located on the dorsal aspect of the vertebral body of S1. A line connecting the midpoint of both circles was generated to represent the iliac axis. As the last step, a second line was drawn extending from the centre point of the cranial circle to superimpose the dorsal plane of the sacrum. The angulation between these two lines represented the sacrum-pelvis angle (Fig. 1). All measurements were performed by a single observer (P.C.).

\section{Statistical analysis}

Data are reported as means and standard deviations (SDs). The assumption of normal distribution was analysed using the Kolmogorov-Smirnov test and was used just for the SPA, not for the NA. The correlation between the SPA and the NA was calculated using Spearman's rank correlation coefficient. The difference in SPA between breeds was calculated using one-way ANOVA followed by post hoc tests using Bonferroni's alpha correction procedure. For the calculation of the difference between males and females within breeds, $t$-tests for independent samples were performed. For all statistical analyses, a $P$ value below 5\% $(P<$ $0.05)$ was considered significant. All statistical analyses were performed by a statistician (A.T.) using IBM SPSS v24 statistical software.

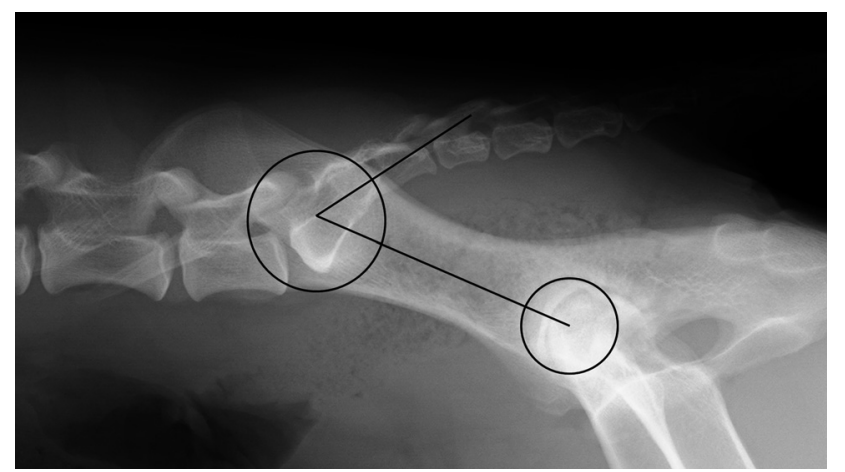

Fig. 1. Representative right lateral lumbosacral radiograph of a dog with the measurement of the sacrum-pelvis angle (SPA), the angulation between the axis of the ilium and the sacrum. First, the axis of the ilium was determined by drawing a circle around the contour of the more cranially located acetabulum and a second circle around the ipsilateral ilium at the height of the sacrum. The second circle was drawn in such a way that it crossed the dorsal and ventral edges of the ilium while the midpoint was positioned on the dorsal surface of the sacrum. The midpoints of the two circles were connected to represent the iliac axis. Second, the angulation between the iliac axis and the sacrum, the SPA, was measured by drawing a line from the midpoint of the second circle to the caudal edge of the sacrum parallel with its dorsal border 
Table 1. The sacrum-pelvis angle (SPA) and the Norberg angle (NA) in different breeds

\begin{tabular}{lccrrr}
\hline \multirow{2}{*}{ Breed } & $\begin{array}{c}\text { Number } \\
\text { of dogs }\end{array}$ & & Min. & Max. & Mean \\
\hline Hungarian Vizsla & 24 & SPA & 48.1 & 67.7 & 59.4 \\
& & NA & 78.9 & 109.0 & 97.8 \\
Rottweiler & \multirow{2}{*}{30} & SPA & 40.0 & 63.4 & 54.6 \\
& & NA & 77.0 & 113.9 & 101.2 \\
Labrador Retriever & \multirow{2}{*}{40} & SPA & 47.7 & 71.4 & 58.6 \\
& & NA & 71.2 & 108.2 & 100.1 \\
Large & \multirow{2}{*}{30} & SPA & 52.6 & 71.5 & 61.4 \\
$\quad$ Münsterländer & & NA & 88.3 & 110.4 & 101.9 \\
Weimaraner & \multirow{2}{*}{34} & SPA & 47.1 & 62.7 & 55.2 \\
& & NA & 82.2 & 110.0 & 99.5 \\
White Shepherd & \multirow{2}{*}{22} & SPA & 43.8 & 63.7 & 53.8 \\
& & NA & 87.9 & 108.2 & 99.6 \\
\hline
\end{tabular}

\section{RESULTS}

The sacrum-pelvis angle (SPA) varied between $40^{\circ}$ and $71.5^{\circ}$ and the Norberg angle (NA) between $71.2^{\circ}$ and $113.9^{\circ}$ (Table 1). The SPA showed high individual variations in each studied breed, the largest in Labrador Retrievers and the smallest in White Shepherds. The largest average angulation was detected in the Large Münsterlander and the smallest in the White Shepherd (Fig. 2).

Based on the one-way ANOVA followed by post hoc tests there was a significant difference in the SPA between some of the breeds, for example between Hungarian Vizsla and Rottweiler, Hungarian Vizsla and Weimaraner, and Hungarian Vizsla and White Shepherd. There was no significant difference between Hungarian Vizsla and Large

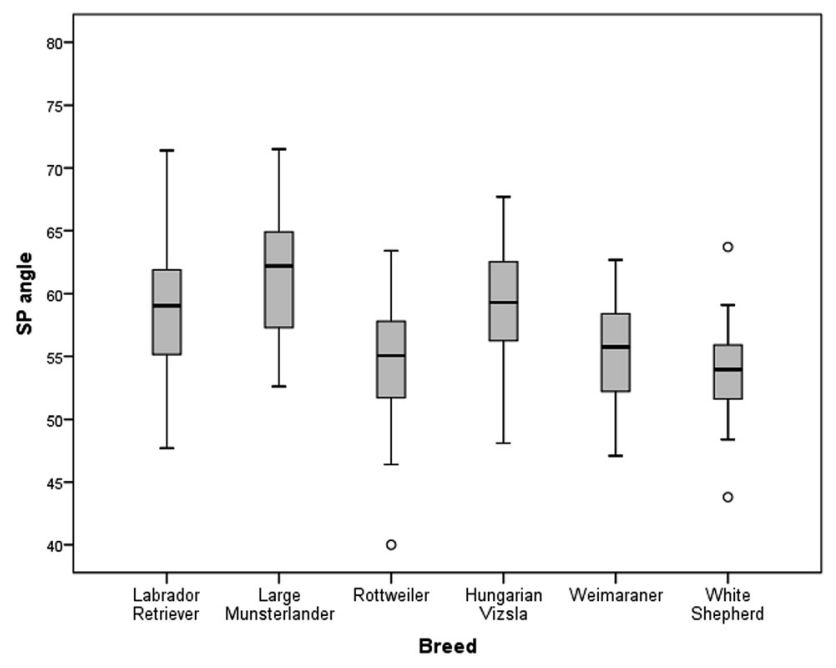

Fig. 2. Sacrum-pelvis angle (SPA) in each breed. Large individual variations and significant breed differences were detected. For example, in the case of the Hungarian Vizsla, a significant difference was detected compared to the Rottweiler, Weimaraner and White Shepherd, but there was no significant difference between the Hungarian Vizsla and the Large Münsterländer and Labrador Retriever
Münsterländer and Labrador Retriever (Table 2). There was no significant difference in the SPA between female and male dogs in any of the breeds (Fig. 3).

According to the Spearman's rank correlation coefficient, there was no significant correlation between the SPA and the $\mathrm{NA}$ in the population of the 180 dogs examined (Fig. 4).

\section{DISCUSSION}

The authors' hypothesis, i.e. that the slope of the pelvis shows significant individual and interbreed variations in dogs, was confirmed by this study. The slope of the pelvis is generally defined as the angulation between the pelvis and the lumbar spine. This angulation can be influenced by some variations in extension or flexion of the lumbosacral region. This may create limitations in reproducibility in measurement of the slope of the pelvis on standard lateral radiographs. Since the sacroiliac joint is a relatively immobile connection between the spine and the pelvis, it is not influenced by the positioning (Evans and de Lahunta, 2013). Therefore, the more consistent and reproducible sacrumpelvis angle (SPA), the angulation between the sacrum and the axis of the ilium was created to represent the differences in the slope of the pelvis. The studied dogs belonged to medium and large breeds, in which hip dysplasia occurs most frequently (Witsberger et al., 2008; Loder and Todhunter, 2017). The available breeds represented a wide range of morphological variation in body conformation from the lightest Vizsla to the heaviest Rottweiler. This constitutional difference is expressed in the differences of the SPA too, because there was a significant difference between some of the breeds. For example, in case of the Hungarian Vizsla, a significant difference was detected compared to Rottweiler, Weimaraner and White Shepherd, but there was no significant difference between Hungarian Vizsla and Large Münsterländer and Labrador Retriever. There were large differences among individuals in each breed, but sex did not affect the SPA. The importance of the slope of the pelvis in the performance of the hind limbs is well known, since it influences the hind limb angulations and standing hind limb postures. In a recent study involving seven different breeds, Doberman Pinschers had the straightest pelvic limbs, while German Shepherds had the most angled pelvic limbs mainly due to the stifle and tarsal joint angulations. The hip angulation, which is influenced by the slope of the pelvis, also plays a role in hind limb angulation, although less than the stifle and tarsal joints (Sabanci et al., 2018). A steep pelvis is advantageous for some purposes, such as quick turning, heavy draft or running uphill, but for speed or endurance on level ground it is a disadvantage. Therefore, the steepness of the pelvis may play an important role in selective dog breeding and can be judged by observing the backward reach of the hind legs while the dog is in motion (Gilbert and Brown, 2001). Our measurements documented high individual and breed variability in the steepness of the pelvis. 
Table 2. The $P$-values of multiple comparisons of the sacrum-pelvis angle

\begin{tabular}{|c|c|c|c|c|c|c|}
\hline Breed & Hungarian Vizsla & Rottweiler & Labrador Retriever & Large Münsterländer & Weimaraner & $\begin{array}{l}\text { White } \\
\text { Shepherd }\end{array}$ \\
\hline Hungarian Vizsla & & 0.005 & 1.000 & 1.000 & 0.019 & 0.02 \\
\hline Rottweiler & 0.005 & & 0.009 & $<0.001$ & 1.000 & 1.000 \\
\hline Labrador Retriever & 1.000 & 0.009 & & 0.272 & 0.037 & 0.003 \\
\hline Large Münsterländer & 1.000 & $<0.001$ & 0.272 & & $<0.001$ & $<0.001$ \\
\hline Weimaraner & 0.019 & 1.000 & 0.037 & $<0.001$ & & 1.000 \\
\hline White Shepherd & 0.002 & 1.000 & 0.003 & $<0.001$ & 1.000 & \\
\hline
\end{tabular}

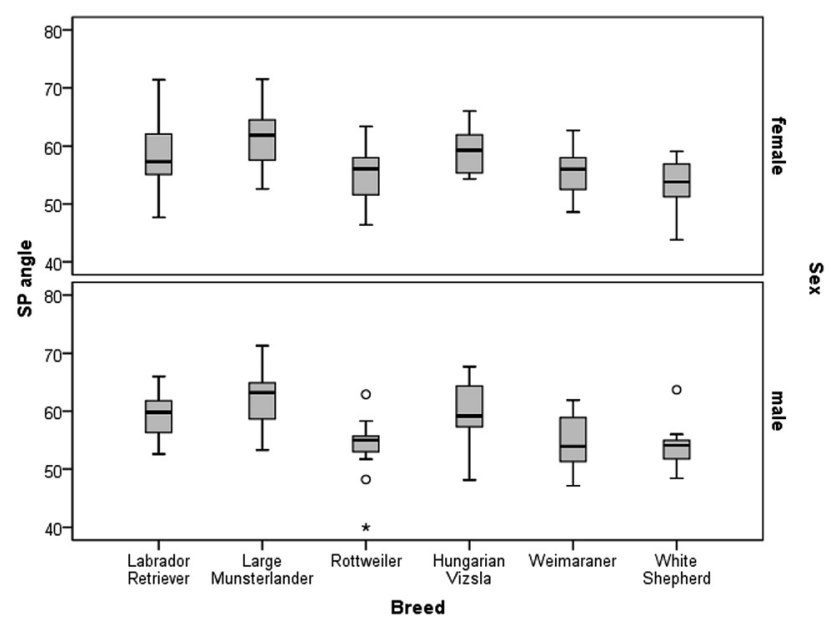

Fig. 3. The sacrum-pelvis angle (SPA) in each breed according to sex. There was no significant sex-related difference in the SPA

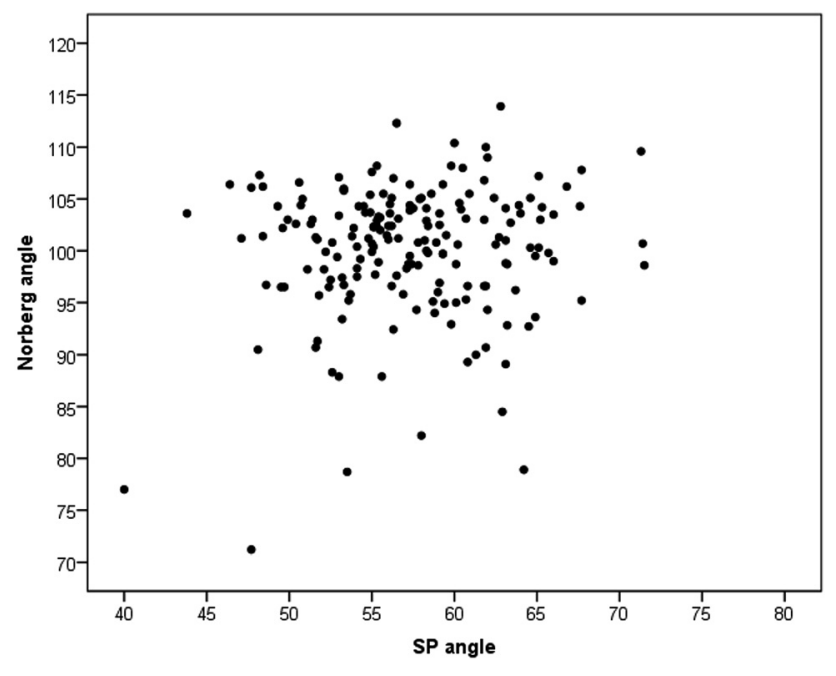

Fig. 4. According to the Spearman's rank correlation coefficient, there was no significant correlation between the sacrum-pelvis angle (SPA) and the Norberg angle (NA) in the population of the 180 dogs examined

The other importance of the slope of the pelvis might be its role in the development of orthopaedic diseases. Some orthopaedic disorders are attributed to variations in pelvic limb posture between dog breeds. For example, a straighter posture of the pelvic limb is suggested as a predisposing factor in the development of stifle disorders, specifically patellar luxation (Bound et al., 2009). Femoral subluxation is considered to be an important factor in CHD (Flückiger et al., 2017). The NA objectively quantifies the degree of femoral subluxation seen on hip-extended radiographs (Henricson et al., 1966). A larger angle indicates a deeper acetabulum and more congruent hips, whereas smaller angles are consistent with increasing degrees of subluxation (Willis, 1997). It shows a significant correlation with the degree of CHD (Henricson et al., 1966; Banfield et al., 1996; Gibbs, 1997; Comhaire and Schoonjans, 2011). It is used in some national and at least in two important international CHD scoring systems: the Fédération Cynologique Internationale (FCI) and the British Veterinary Association/Kennel Club (BVA/KC) in the evaluation of CHD (Gibbs, 1997; Ginja et al., 2010; Comhaire and Schoonjans, 2011). It is concluded that precise measurement of the NA using a system of digital image analysis and the calculation of percentile rank order by breed may contribute to the evaluation of hip radiographs for $\mathrm{CHD}$, and also to the reduction of the prevalence of this condition among pedigree dogs (Comhaire and Schoonjans, 2011). The NA is not used as a single parameter in the radiographic evaluation of $\mathrm{CHD}$, but in the authors' opinion it is adequate for detecting tendentious changes in the correlation of femoral head subluxation and slope of the pelvis. In our study, the NA was correlated with the SPA as a representative of the differences in the slope of the pelvis. According to our measurements of 180 dogs, there was no correlation between the SPA and the NA. Often similar NAs were measured when the sacrumpelvis angles were different. Therefore, a tendentious correlation between flat or steep pelvic conformation and femoral subluxation could not be proven. This suggests that the slope of the pelvis does not play a significant role in CHD.

The study has some limitations. It would be ideal to record the SPA for more breeds with bigger morphological variations. The studied breeds were those in which standard ventrodorsal hip and lateral lumbosacral radiographs were available in our archive in a number sufficient for the statistical analysis. Since most of the examined dogs were young breeding dogs, some preselection of the sample population cannot be ruled out. In the authors' opinion, such a preselection did not influence significantly the correlation test between the SPA and the NA. A probable limitation is related to the measurement technique of the NA because of the possible effect of hip extension on joint laxity. It is a generally accepted assumption that in ventrodorsal position with the extension of pelvic limbs, the 
femoral head is pushed inside the acetabulum (Skurkova et al., 2010). Another radiographic possibility to estimate the degree of passive laxity of the hip joint is the distractionstress radiograph, which is considered to be more sensitive than the NA (Smith et al., 2012). We could not use this technique for our measurements, because distraction-stress radiography was not available in sufficient numbers in our archive. However, in the authors' opinion, the somewhat higher sensitivity of distraction-stress radiography would not have changed the outcome of the correlation study.

In conclusion, the findings indicate that there are significant individual and interbreed variations in the slope of the pelvis. No significant relationship between the slope of the pelvis and femoral subluxation could be identified, however.

\section{ACKNOWLEDGEMENT}

The study was presented as an oral presentation at the 23rd European Veterinary Diagnostic Imaging Annual Conference, Basel, Switzerland, 2019.

\section{REFERENCES}

Banfield, C. M., Bartels, J. E., Hudson, J. A., Wright, J. C., Hathcock, J. T. and Montgomery, R. D. (1996): A retrospective study of canine hip dysplasia in 116 military working dogs. Part I: angle measurements and orthopedic foundation for animals (OFA) grading. J. Am. Anim. Hosp. Assoc. 32, 413-422.

Bound, N., Zakai, D., Butterworth, S. J. and Pead, M. (2009): The prevalence of the canine patellar luxation in three centres. Clinical features and radiographic evidence of limb deviation. Vet. Comp. Orthop. Traumatol. 22, 32-37.

Comhaire, F. H. and Schoonjans, F. A. (2011): Canine hip dysplasia: The significance of the Norberg angle for healthy breeding. J. Small Anim. Pract. 52, 536-542.

Evans, H. E. and de Lahunta, A. (2013): Miller's Anatomy of the Dog, 4th ed. Saunders, Philadelphia. 176 pp.

Fealey, M. J., Li, J., Todhunter, R. J. E., Krotscheck, U., Hayashi, K., McConkey, M. J., Boyko, A. R., Hayward, J. J. and Todhunter, R. J. (2017): Genetic mapping of principal components of canine pelvic morphology. Canine Genet. Epidemiol. 24, 4.

Flückiger, M. A. (2007): How to take and read hip joint radiographs in a structured way. Eur. J. Comp. Anim. Pract. 17, 133-134.

Flückiger, M. A., Steffen, F., Hassig, M. and Morgan, J. P. (2017): Asymmetrical lumbosacral transitional vertebrae in dogs may promote asymmetrical hip joint development. Vet. Comp. Orthop. Traumatol. 30, 137-142.
Gibbs, C. (1997): The BVA/KC scoring scheme for control of hip dysplasia: interpretation of criteria. Vet. Rec. 141, 275-284.

Gilbert, E. M. and Brown, T. R. (2001): K-9 Structure and Terminology. Dogwise Publishing, Washington. pp. 74-89.

Ginja, M. M. D., Silvestre, A. M., Gonzalo-Orden, J. M. and Ferreira, A. J. (2010): Diagnosis, genetic control and preventive management of canine hip dysplasia: A review. Vet. J. 184, 269276.

Henricson, B., Norberg, I. and Olsson, S. E. (1966): On the etiology and pathogenesis of hip dysplasia: A comparative review. J. Small Anim. Pract. 7, 673-688.

Kealy, R. D., Olsson, S. E., Monti, K. L., Lawler, D. F., Biery, D. N., Helms, R. W., Lust, G. and Smith, G. K. (1992): Effects of limited food consumption on the incidence of hip dysplasia in growing dogs. J. Am. Vet. Med. Assoc. 201, 857-863.

Loder, R. T. and Todhunter, R. J. (2017): The demographics of canine hip dysplasia in the United States and Canada. J. Vet. Med. 2017, 5723476.

Lust, G. (1997): An overview of the pathogenesis of canine hip dysplasia. J. Am. Vet. Med. Assoc. 210, 1443-1445.

Nickel, R., Schummer, A., Seiferle, E., Frewein, J., Wilkens, H. and Wille, K. H. (1986): The Locomotor System of the Domestic Mammals. Verlag Paul Parey, Berlin. 52 pp.

Sabanci, S. S. and Ocal, M. K. (2018): Categorization of the pelvic limb standing posture in nine breeds of dogs. Anat. Histol. Embryol. 47, 58-63.

Sarierler, M. (2004): Comparison of femoral inclination angle measurements in dysplastic and nondysplastic dogs of different breeds. Acta Vet. Hung. 52, 245-252.

Skurkova, L., Hluchy, M., Lackova, M., Hihalova, M. and Ledecky, V. (2010): Relation of the Norberg angle and position of the femoral head centre to the dorsal acetabular edge in evaluation of canine hip dysplasia. Vet. Comp. Orthop. Traumatol. 23, 433-438.

Smith, G. K., Karbe, G. T., Agnello, K. A. and McDonald-Lynch, M. B. (2012): Pathogenesis, diagnosis, and control of canine hip dysplasia In: Tobias, K. M. and Johnson, S.A. (eds.) Veterinary surgery: Small animal, vol. 1, 1st ed. Saunders/Elsevier, St. Louis (MO). pp. 824-848.

Smith, G. K., Popovitch, C.A., Gregor, T. P. and Schofer, F. S. (1995): Evaluation of risk factors for degenerative joint disease associated with hip dysplasia in dogs. J. Am. Vet. Med. Assoc. 206, 642-647.

Willis, M. B. (1997): A review of the progress in canine hip dysplasia control in Britain. J. Am. Vet. Med. Assoc. 210, 14801482.

Witsberger, T. H., Villamil, J. A., Schultz, L. G., Hahn, A. W. and Cook, J. L. (2008): Prevalence of and risk factors for hip dysplasia and cranial cruciate ligament deficiency in dogs. J. Am. Vet. Med. Assoc. 232, 1818-1824. 